\title{
Association between acrylamide hemoglobin adduct levels and depressive symptoms in US adults: NHANES 2013-2016.
}

Zhaoying Li ${ }^{\S}$, Jing Sun ${ }^{\S}$, Dongfeng Zhang ${ }^{\S *}$.

§: Department of Epidemiology and Health Statistics, the College of Public Health of Qingdao University, 308 Ningxia Road, Qingdao, Shandong 266071, People's Republic of China.

${ }^{*}$ Corresponding author at: Department of Epidemiology and Health Statistics, the College of Public Health of Qingdao University, 308 Ningxia Road, Qingdao, Shandong 266071, China;

E-Mail addresses: zhangdf1961@126.com . 
Table S1. Weighted ORs and $95 \%$ CIs for depressive symptoms according to HbAA,

$\mathrm{HbGA}$, and $\mathrm{HbGA} / \mathrm{HbAA}$ as continue variable.

\begin{tabular}{llll}
\hline & Crude & Model 1 $^{\mathbf{a}}$ & Model 2 $^{\mathbf{b}}$ \\
\cline { 2 - 4 } & OR (95\% CI) & OR (95\% CI) & OR (95\% CI) \\
\hline HbAA $^{\mathbf{c}}$ & $1.004(1.002,1.006)^{*}$ & $1.004(1.002,1.006)^{* *}$ & $1.006(1.000,1.013)^{*}$ \\
HbGA $^{\mathbf{d}}$ & $1.004(1.002,1.007)^{*}$ & $1.005(1.002,1.008)^{*}$ & $0.991(0.981,1.000)$ \\
HbGA/HbAA $^{*}$ & $0.402(0.184,0.878)^{*}$ & $0.336(0.153,0.738)^{*}$ & $0.429(0.165,1.111)$ \\
\hline
\end{tabular}

${ }^{a}$ : model 1 were adjusted by age, and sex; ${ }^{b}$ : model 2 were adjusted by age, sex, BMI, race, education, household income, marital status, and carbohydrate in energy density, caffeine intake, serum cotinine level, alcohol use, physical activity, hypertension, diabetes, antidepressants use; $c$ : additionally adjust HbGA in model $2{ }^{d}$ : additionally adjust HbAA in model $2 .{ }^{*}: P<0.05 * *$ : $P<0.01$; OR, odds ratio; CI, confidence interval; 
Table S2. The interaction between covariates and HbAA, HbGA, HbGA/HbAA.

\begin{tabular}{|c|c|c|c|}
\hline$P$ for interaction a & HbAA & HbGA & HbGA/HbAA \\
\hline Sex & 0.19 & 0.2 & 0.36 \\
\hline Age & 0.22 & 0.14 & 0.14 \\
\hline Race & 0.23 & $0.04 *$ & 0.23 \\
\hline Education & 0.35 & 0.43 & 0.69 \\
\hline Household income & 0.11 & $0.05^{*}$ & 0.13 \\
\hline Marital status & 0.93 & $0.02 *$ & 0.28 \\
\hline BMI & 0.82 & 0.05 & 0.48 \\
\hline Drinking Status & 0.37 & $0.01 *$ & 0.33 \\
\hline Physical activity & 0.29 & 0.24 & 0.4 \\
\hline Hypertension & 0.33 & 0.11 & 0.77 \\
\hline Diabetes & 0.47 & 0.27 & 0.45 \\
\hline Antidepressants use & 0.84 & 0.92 & 0.86 \\
\hline Carbohydrate energy density & 0.97 & 0.376 & 0.38 \\
\hline Caffeine & 0.06 & 0.27 & $0.04 *$ \\
\hline Cotinine & 0.72 & 0.09 & 0.68 \\
\hline
\end{tabular}

a, All the results were conducted in final model.

${ }^{*} P<0.05$ 
Table S3. Weighted ORs and 95\%CIs for depressive symptoms by subgroup of sex according to quartiles of $\mathrm{HbAA}, \mathrm{HbGA}$, and $\mathrm{HbGA} / \mathrm{HbAA}$.

\begin{tabular}{|c|c|c|c|c|c|}
\hline & \multirow{2}{*}{ Cutoff(pmol/g) } & \multirow{2}{*}{$\begin{array}{l}\text { Cases/ } \\
\text { Participants }\end{array}$} & \multirow{2}{*}{$\begin{array}{l}\text { Crude } \\
\text { OR }(95 \% \text { CI })\end{array}$} & \multirow{2}{*}{$\begin{array}{l}\text { Model 1 }^{a} \\
\text { OR }(95 \% \text { CI })\end{array}$} & \multirow{2}{*}{$\begin{array}{l}\text { Model } 2^{b} \\
\text { OR }(95 \% \text { CI })\end{array}$} \\
\hline & & & & & \\
\hline \multicolumn{6}{|c|}{ Male } \\
\hline \multicolumn{6}{|c|}{$\mathbf{H b A A}^{c}$} \\
\hline Q1 & $<32.50$ & $21 / 332$ & Ref. & Ref. & Ref. \\
\hline Q2 & 32.50 to $<42.50$ & $21 / 369$ & $0.67(0.27,1.68)$ & $0.70(0.28,1.75)$ & $0.50(0.11,2.29)$ \\
\hline Q3 & 42.50 to $<63.25$ & $32 / 403$ & $0.93(0.39,2.20)$ & $0.94(0.39,2.24)$ & $1.62(0.57,4.58)$ \\
\hline Q4 & $\geq 63.25$ & $39 / 475$ & $1.70(0.73,3.94)$ & $1.70(0.72,4.00)$ & $2.74(0.66,11.37)$ \\
\hline \multicolumn{6}{|c|}{ HbGA $^{d}$} \\
\hline Q1 & $<27.50$ & $25 / 379$ & Ref. & Ref. & Ref. \\
\hline Q2 & 27.50 to $<37.80$ & $23 / 368$ & $0.53(0.20,1.41)$ & $0.55(0.21,1.49)$ & $0.71(0.24,2.07)$ \\
\hline Q3 & 37.80 to $<54.00$ & $20 / 376$ & $0.57(0.23,1.43)$ & $0.61(0.24,1.52)$ & $0.73(0.23,2.28)$ \\
\hline Q4 & $\geq 54.00$ & $37 / 407$ & $1.23(0.57,2.69)$ & $1.31(0.59,2.89)$ & $1.14(0.21,6.18)$ \\
\hline \multicolumn{6}{|c|}{ HbGA/HbAA } \\
\hline Q1 & $<0.68$ & $32 / 465$ & Ref. & Ref. & Ref. \\
\hline Q2 & 0.68 to $<0.82$ & $25 / 383$ & $0.73(0.40,1.36)$ & $0.78(0.42,1.44)$ & $0.68(0.31,1.51)$ \\
\hline Q3 & 0.82 to $<0.98$ & $21 / 348$ & $0.71(0.34,1.48)$ & $0.80(0.38,1.71)$ & $0.85(0.28,2.56)$ \\
\hline Q4 & $\geq 0.98$ & $23 / 276$ & $0.79(0.40,1.57)$ & $0.96(0.48,1.92)$ & $1.38(0.64,3.00)$ \\
\hline \multicolumn{6}{|c|}{ Female } \\
\hline \multicolumn{6}{|c|}{$\mathbf{H b A A}^{c}$} \\
\hline Q1 & $<32.50$ & $40 / 435$ & Ref. & Ref. & Ref. \\
\hline Q2 & 32.50 to $<42.50$ & $25 / 425$ & $0.50(0.22,1.12)$ & $0.55(0.26,1.15)$ & $0.66(0.25,1.69)$ \\
\hline Q3 & 42.50 to $<63.25$ & $25 / 395$ & $0.67(0.32,1.40)$ & $0.73(0.35,1.50)$ & $0.84(0.31,2.29)$ \\
\hline Q4 & $\geq 63.25$ & $65 / 323$ & $2.92(1.58,5.42)^{*}$ & $3.37(1.90,5.98)^{*}$ & $1.84(0.68,5.02)$ \\
\hline \multicolumn{6}{|c|}{ HbGA $^{d}$} \\
\hline Q1 & $<27.50$ & $33 / 352$ & Ref. & Ref. & Ref. \\
\hline Q2 & 27.50 to $<37.80$ & $30 / 398$ & $0.83(0.45,1.51)$ & $0.76(0.42,1.39)$ & $0.79(0.39,1.61)$ \\
\hline Q3 & 37.80 to $<54.00$ & $25 / 404$ & $0.49(0.29,0.81)^{*}$ & $0.44(0.25,0.77)^{*}$ & $0.43(0.21,0.92)^{*}$ \\
\hline Q4 & $\geq 54.00$ & $63 / 357$ & $2.70(1.65,4.41)^{*}$ & $2.44(1.36,4.40)^{*}$ & $1.32(0.56,3.11)$ \\
\hline \multicolumn{6}{|c|}{ HbGA/HbAA } \\
\hline Q1 & $<0.68$ & $41 / 267$ & Ref. & Ref. & Ref. \\
\hline Q2 & 0.68 to $<0.82$ & $37 / 340$ & $0.40(0.22,0.74)^{*}$ & $0.40(0.21,0.75)^{*}$ & $0.53(0.28,1.03)$ \\
\hline Q3 & 0.82 to $<0.98$ & $32 / 388$ & $0.45(0.26,0.78)^{*}$ & $0.39(0.21,0.72)^{*}$ & $0.54(0.26,1.12)$ \\
\hline Q4 & $\geq 0.98$ & $37 / 459$ & $0.44(0.25,0.78)^{*}$ & $0.34(0.18,0.63)^{*}$ & $0.62(0.28,1.37)$ \\
\hline
\end{tabular}

${ }^{a}$ : model 1 were adjusted by age, and BMI; ${ }^{b}$ : model 2 were adjusted by age, BMI, race, education, household income, marital status, and carbohydrate in energy density, caffeine intake, serum cotinine level, alcohol use, physical activity, hypertension, diabetes, antidepressants use; $c$ : additionally adjust HbGA in model 2 ; ${ }^{d}$ : additionally adjust HbAA in model $2 .{ }^{*}: P<0.05 * *$ : $P<0.01$; OR, odds ratio; CI, confidence interval; 
Table S4. Sensitivity analyses of ORs and 95\%CIs of AA biomarkers and depressive symptoms.

\begin{tabular}{|c|c|c|c|c|c|c|c|}
\hline & \multirow{2}{*}{ Cutoff(pmol/g) } & \multicolumn{2}{|c|}{ Exclude extreme biomarkers $^{a}$} & \multicolumn{2}{|c|}{ Exclude extreme BMI ${ }^{a}$} & \multicolumn{2}{|c|}{ Exclude extreme energy $^{a}$} \\
\hline & & Case/N & OR(95\% CI) & Case/N & OR(95\% CI) & Case/N & OR(95\% CI) \\
\hline \multicolumn{8}{|c|}{ HbAA (pmol/g Hb) } \\
\hline Q1 & $<32.50$ & $56 / 739$ & Ref. & $56 / 744$ & Ref. & $60 / 753$ & Ref. \\
\hline Q2 & 32.50 to $<42.50$ & $46 / 794$ & $0.61(0.33,1.14)$ & $42 / 778$ & $0.55(0.29,1.06)$ & $46 / 785$ & $0.58(0.31,1.08)$ \\
\hline Q3 & 42.50 to $<63.25$ & $57 / 798$ & $1.07(0.44,2.59)$ & $55 / 789$ & $1.06(0.44,2.56)$ & $55 / 783$ & $1.07(0.45,0.26)$ \\
\hline Q4 & $\geq 63.25$ & $100 / 767$ & $2.38(1.18,4.82)^{*}$ & $104 / 791$ & $2.53(1.29,4.97)^{*}$ & $101 / 775$ & $2.54(1.29,5.01)^{*}$ \\
\hline \multicolumn{8}{|c|}{ HbGA (pmol/g Hb) } \\
\hline Q1 & $<27.50$ & $52 / 698$ & Ref. & $55 / 711$ & Ref. & $58 / 722$ & Ref. \\
\hline Q2 & 27.50 to $<37.80$ & $53 / 766$ & $0.78(0.47,1.31)$ & $49 / 755$ & $0.74(0.43,1.28)$ & $53 / 751$ & $0.76(0.44,1.31)$ \\
\hline Q3 & 37.80 to $<54.00$ & $45 / 780$ & $0.52(0.26,1.05)$ & $43 / 768$ & $0.51(0.25,1.07)$ & $45 / 770$ & $0.53(0.25,1.10)$ \\
\hline Q4 & $\geq 54.00$ & $100 / 737$ & $1.11(0.48,2.57)$ & $98 / 755$ & $1.28(0.56,2.93)$ & $96 / 740$ & $1.22(0.53,2.82)$ \\
\hline \multicolumn{8}{|c|}{ HbGA/HbAA } \\
\hline Q1 & $<0.68$ & $70 / 703$ & Ref. & $72 / 724$ & Ref. & $72 / 715$ & Ref. \\
\hline Q2 & 0.68 to $<0.82$ & $62 / 723$ & $0.64(0.38,1.05)$ & $59 / 709$ & $0.61(0.37,0.99)^{*}$ & $61 / 708$ & $0.60(0.37,0.97)^{*}$ \\
\hline Q3 & 0.82 to $<0.98$ & $53 / 736$ & $0.55(0.32,0.95)^{*}$ & $51 / 721$ & $0.56(0.33,0.94)^{*}$ & $53 / 723$ & $0.54(0.32,0.90)^{*}$ \\
\hline Q4 & $\geq 0.98$ & $55 / 707$ & $0.75(0.42,1.33)$ & $56 / 722$ & $0.70(0.39,1.28)$ & $58 / 724$ & $0.69(0.39,1.23)$ \\
\hline
\end{tabular}

a: Principles of exclusion: $>99 \%$ percentile, or $<1 \%$ percentile of the samples. Adjusted covariates: age, sex, BMI, race, marital status, education, annual household income, carbohydrate in energy density, caffeine intake, serum cotinine level, alcohol use, physical activity, hypertension, diabetes, antidepressants use. Additionally, HbAA and HbGA were adjusted with each other. OR: odds ratio. CI: confidence interval. 